\title{
Unaffected microscopic dynamics of macroscopically arrested water in dilute clay gels
}

\author{
Tilo Seydel, ${ }^{1, *}$ Lutz Wiegart, ${ }^{2}$ Fanni Juranyi, ${ }^{3}$ Bernd Struth, ${ }^{4}$ and Helmut Schober ${ }^{1}$ \\ ${ }^{1}$ Institut Max von Laue-Paul Langevin, Boîte Postale 156, F-38042 Grenoble Cedex 9, France \\ ${ }^{2}$ European Synchrotron Radiation Facility, Boîte Postale 220, F-38043 Grenoble Cedex 9, France \\ ${ }^{3}$ Labor für Neutronenstreuung, ETH Zürich \& PSI, CH-5232 Villigen PSI, Switzerland \\ ${ }^{4}$ HASYLAB am DESY, Notkestraße 85 D-22603 Hamburg, Germany
}

(Received 2 September 2008; published 18 December 2008)

\begin{abstract}
Adequate clay minerals considerably affect the macroscopic mechanical behavior of water even at concentrations of a few percent. Thus when 2 wt. \% laponite clay mineral nanoparticles are added to water, the resulting colloidal suspension after some time takes on the semisolid characteristics of a jellylike material at room temperature. Cold neutron time-of-flight spectroscopy data are in agreement with the assumption that notwithstanding this macroscopic change, the mobility of the water molecules on intermolecular and intramolecular length scales remains largely unaffected. This observation is discussed in the context of the properties and the role of water in different more or less dilute ionic environments. The result contributes to the ongoing debate of the properties and role of water in living cells.
\end{abstract}

DOI: 10.1103/PhysRevE.78.061403

PACS number(s): 82.70.- y, 83.80.Hj, 83.80.Kn

\section{INTRODUCTION}

The interaction between water and charged particles is an issue at the center of understanding biological processes [1]. Biological systems comprise charged particles and complex macromolecules such as proteins in aqueous solution as essential constituents. A hypothesis has been proposed that at the origin of life the first cells assembled in a hydrogel environment [2]. According to this hypothesis the ensemble of charged particles, macromolecules, and water is assumed to form a gel which essentially defines the cell, and a gel-like precursor assembly of a cell can function on a primitive basis without the necessity of the presence of a cell membrane. More generally, it is assumed that the biological function of proteins and other intracellular macromolecules cannot be understood without taking into account the mobility of the water molecules surrounding them in vivo (Ref. [1], and references therein). Further, charges in the aqueous solution have an influence on the biological function of proteins [3]. In the extreme case, charges induced by salts can for instance cause the denaturing of proteins in aqueous solutions. On larger length scales, different organelles such as mitochondria may be assumed to affect the water mobility in intracellular environments. A current discussion in cell biophysics addresses the question whether the dynamics of cell water is differently affected by the intracellular environment on micrometric and atomic length scales [4]. Whether or not water is slowed down significantly on atomic length scales in biological cells and whether such an effect, if present, would affect the biological function, is at the center of the debate $[5,6]$. For the sole purpose of looking for some inspiration in this biological and possibly other contexts of soft matter systems, here we report on a study of a clay mineral hydrogel system that has no immediate relation with protein solutions or entire biological cells. The conclusions from our study on this system have, however, an interesting resemblance to

\footnotetext{
*seydel@ill.eu
}

some conclusions drawn in Ref. [4] on the properties of Escheria coli intracellular water, while putting some of the interpretations made therein in a different perspective.

Gelation phenomena can be observed in colloidal as well as in polymer systems [7]. Gels, in general, can be defined as being either colloidal suspensions of mesocopic solid particles in a liquid or solutions of polymeric molecules, in both cases forming a jellylike material. In our study we focus on a model colloidal gel. Gels have an elastic modulus caused by a gelator network which can immobilize large amounts (up to 99 wt. \%) of liquid on a macroscopic scale (Fig. 1, top). In rheological terms, for a gel the absolute value of the tensile storage modulus is greater than the absolute value of the loss modulus. Gels can emerge over time from the so-called sol state, the sol being the precursor colloidal suspension (or polymer solution for a polymer sol) with properties resembling those of a viscous liquid (Fig. 1, bottom). The sol-gel transition (gel point) is the stage at which a liquid begins to take on the semisolid characteristics of a gel. The sol-gel transition is accompanied by a divergence of the viscosity and appearance of the elastic modulus due to the buildup of the gelator network. Remarkably, this transition may take place at ambient temperature solely dependent on the concentration of the suspension. This transition from a macro-

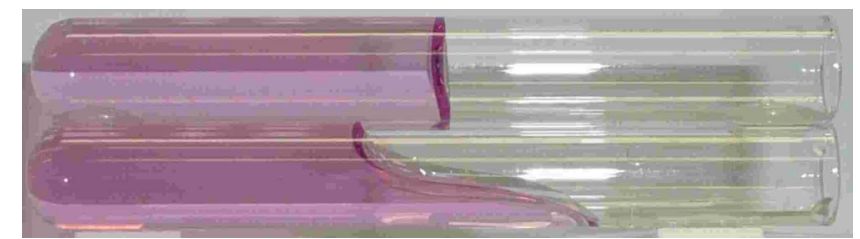

FIG. 1. (Color online) Photograph of a suspension of 2 wt. \% laponite nanoparticles in water (top), and of a suspension of 1.2 wt. \% laponite in water (bottom). The samples were colored with $\mathrm{KMnO}_{4}$ for better visibility. The direction of gravity being perpendicular to the test tube cylinder axis, the photograph illustrates that the $2 \mathrm{wt}$ \% suspension forms a semisolid material (gel), while the 1.2 wt. \% suspension remains in a highly viscous (sol) state on the time scale of our experiment. 
scopically fluid into a macroscopically arrested material therefore differs from a glass transition in atomic or molecular glass formers where a microscopically nonergodic glass state is achieved by an adequate cooling. We note that the definition of the glass state is still being discussed for colloidal systems [8].

As a model system we have investigated aqueous clay mineral colloidal gels $[9,10]$, which, in addition to their fundamental interest, are also of practical use in industry $[11,12]$, for instance, as rheology modifiers in products ranging from hair styling sprays to tire surface protectants [13]. The clay mineral gelator used in our experiment was the industrially fabricated synthetic layered silicate laponite with the shape of a nanodisk with a thickness of $9.5 \AA$ and a mean diameter of $250 \AA$. The surface of the nanodisks is negatively charged and their edges positively with an overall electron excess of 0.7 electrons/unit cell. This electron excess is counterbalanced by sodium ions. Dry laponite consists mainly of $\mathrm{SiO}_{2}$ (nearly 60 wt. \%) and $\mathrm{MgO}$ (nearly 28 wt. \%) and contains some $\mathrm{Na}_{2} \mathrm{O}$ and $\mathrm{Li}_{2} \mathrm{O}$. In our experiment the thixotropic gels have been obtained by dispersing 2.0 wt. \% laponite powder in ultrapure water. For the resulting colloidal suspension of laponite nanoparticles in water the sol-gel transition is reversible, i.e., the stiff gel emerging with time can be returned to the sol state through sufficient mechanical stirring. Neutron small-angle scattering experiments on clay mineral gels have already been reported [14], and the advantage of the use of neutrons is in the very low interaction with the minerals. The neutrons thus probe predominantly the water molecules.

Other remarkable properties of such diluted clay mineral gel systems affect the surfaces of these gels. The interface between a gel and bulk water remains stable for an arbitrarily long time, provided that the bulk water is added carefully and the gel remains mechanically undisturbed. Water and certain ions such as the permanganate ion of $\mathrm{KMnO}_{4}$ nevertheless diffuse freely past this interface [15]. The surface of a gel may be as smooth as a free water surface [16,17]. Water can evaporate from a gel surface very similarly to a free water surface.

The gel state is assumed to be related to electrostatic interactions between the charged disks. The gel structure has been tentatively explained using a "house-of-cards" network model $[9,18]$. Alternative models in the ongoing study of gel formation mechanisms [19] employ electrostatic repulsions between the clay particles [10] or discuss clay gels in terms of a liquid crystal [20]. However, the microscopic dynamics of water confined to such a gel structure is to our knowledge not yet fully explored. The investigation of water dynamics in a gel system raises a series of novel issues as it combines fundamental aspects of complex fluids and dynamics in confinement. With view at the macroscopic observations on aqueous gels, a change of the water dynamics in sols and gels might be expected. Thus, NMR experiments show that the long-range diffusion of the water molecules is slowed down with rising concentration of the mineral suspension [21]. These experiments access large length scales, averaging over more than $15 \mu \mathrm{m}$ in the case of Ref. [21]. Further, dynamic light scattering (DLS) experiments have been reported indicating that very slow processes leading to gelation can be observed even in highly dilute laponite gels [22]. X-ray photon correlation spectroscopy (XPCS) experiments on these systems have also been reported [23], addressing aging effects in the gels. Depending on the mineral concentration, the time scale for the gel to reach its nonergodic stable state can thus vary from hours to months, as has been investigated by a combination of small-angle x-ray scattering and DLS [24]. However, these studies report experiments predominantly sensitive to mesoscopic length scales where the "compartment size" within the gels according to the "house-of-cards" model may play a role. In addition, the NMR experiments indicate noticeable effects only at relatively high mineral concentrations (measurable deviations from the diffusion of pure water having been reported for laponite concentrations in water of $3 \mathrm{wt} . \%$ and higher [21]). We therefore wish to extend this information by the investigation of faster motions on shorter length scales.

As we will discuss in the following sections, our results using neutron time-of-flight spectroscopy indicate that no significant dynamical change occurs in dilute aqueous gels on the time scales of molecular jump diffusive motion on atomic length scales, as compared to pure bulk water. There is only a small density effect with rising mineral concentration visible in the neutron scattering signal.

\section{EXPERIMENTAL: MATERIALS AND METHODS}

We have carried out a series of experiments on cold neutron time-of-flight (IN5 and IN6 at ILL, FOCUS at SINQ/ PSI) and backscattering spectrometers (IN10 at ILL) to check the reliability and reproducibility of the results. We have thus covered a scattering vector range of approximately $0.2 \AA^{-1} \leqslant Q \leqslant 2 \AA^{-1}$, and neutron energy transfer ranges of approximately $-16 \mu \mathrm{eV} \leqslant \hbar \omega \leqslant 16 \mu \mathrm{eV}$ at $\approx 1 \mu \mathrm{eV}$ FWHM resolution, and $-1 \mathrm{meV} \leqslant \hbar \omega \leqslant 20 \mathrm{meV}$ at $\approx 100 \mu \mathrm{eV}$ FWHM resolution using the backscattering and time-offlight techniques, respectively. The data shown in the present report have been recorded at FOCUS [25] (FOCUS run II/ 04, 002149++) using an incident wavelength $\lambda=5.1 \AA$ (FWHM of the nearly Gaussian, $Q$-dependent resolution function at $Q=1 \AA^{-1}$ approximately $95 \mu \mathrm{eV}$ ), and an approximately $3 \mathrm{~cm}$ wide and $5 \mathrm{~cm}$ high incident beam. The resolution was measured by a vanadium foil (mass $13 \mathrm{~g}$, height $5.5 \mathrm{~cm}$ ) inside the outer wall of the sample container. The minimum accessible scattering vector at FOCUS in the chosen setup is $Q \approx 0.25 \AA^{-1}$. The sample was kept in a standard Orange cryostat at the temperature $T=285 \mathrm{~K}$.

Laponite RD® was obtained from Rockwood Additives, Ltd. [13]. The laponite clay mineral concentration was adjusted to 2 wt. \% for the gel, and the gel was prepared ex situ from ultrapure $\mathrm{H}_{2} \mathrm{O}$ (purifying apparatus: ELGA PURELAB classic) in a glass jar two days prior to the experiment, assuring a good apparent solidlike rigidity. Analogously, a sol sample was prepared by using only $1.2 \mathrm{wt}$ \% laponite minerals (for a phase diagram of laponite in aqueous solution see, e.g., Ref. [26]). This sample had a highly viscous, however, not fully rigid appearence, and was thus in the sol state on the time scale of the experiment. The sol and gel samples were prepared by dispersing the laponite powder in ultrapure 
water followed by rapid vortex stirring for about $1 \mathrm{~h}$. The samples were prepared in an air-conditioned chemistry laboratory at ambient air, and the glass jars were sealed subsequent to the sample preparation. The excess gel and sol not filled in the sample container was kept in the original glass jar until the end of the experiment to verify that the samples remained in the gel and sol states, respectively, for the duration of the experiment. As a reference, a sample of pure water has in addition been measured to compare with the existing data on water (see, e.g., Refs. [27-29], and references therein). We have measured the mass density of water, gels and sols using a PAAR DMA 58 densimeter. We have obtained the following mass densities of reference pure water; 1.2 wt. $\%$ sol and 2 wt. $\%$ gel samples, respectively, at $T=12.5^{\circ} \mathrm{C}$ using this densimeter: $\rho_{\mathrm{H}_{2} \mathrm{O}}=(0.9994 \pm 5$ $\left.\times 10^{-5}\right) \mathrm{g} / \mathrm{cm}^{3}, \quad \rho_{\mathrm{sol}}=\left(1.0061 \pm 5 \times 10^{-5}\right) \mathrm{g} / \mathrm{cm}^{3}, \quad \rho_{\text {gel }}$ $=\left(1.0100 \pm 5 \times 10^{-5}\right) \mathrm{g} / \mathrm{cm}^{3}$. The dissolved laponite particles themselves have a mass density of $(2.42 \pm 0.12) \mathrm{g} / \mathrm{cm}^{3}[30]$.

When handling gels in aluminum sample containers, difficulties arise due to the alkaline property of the laponite gels ( $\mathrm{pH} 9.8$ for a 2 wt. \% suspension [13]). The aluminum sample container material thus corrodes by the reaction $2 \mathrm{Al}+6 \mathrm{H}_{2} \mathrm{O} \rightarrow 2 \mathrm{Al}(\mathrm{OH})_{3}+3 \mathrm{H}_{2}$, and the resulting gas pressure deforms the containers. In order to circumvent this difficulty, we employed an anodized aluminum container and we adjusted the sol and gel samples to a neutral $\mathrm{pH}$ value using citric acid (Sigma Aldrich, anhydrous, $\geqslant 99.5 \%, \mathrm{CAS}$ : 77-92-9). The neutral $\mathrm{pH}$ ( $\mathrm{pH} 7.2)$ of the samples was adjusted during the preparation of the laponite suspensions. The citric acid was thus added during the vortex stirring of the suspensions after a full dispersion and hydration of the laponite had been reached, i.e., when the supension had become transparent. The use of citric acid to obtain sols and gels, respectively, with a neutral $\mathrm{pH}$ is a standard method described by the manufacturer of laponite [31]. We have verified by rheology measurements that the $2 \mathrm{wt}$. \% laponite samples with the neutral $\mathrm{pH}$ are indeed in the gel state [15]. The absolute values and the frequency response of their storage and loss moduli thus resemble to $2 \mathrm{wt}$. \% laponite gel at $\mathrm{pH} 10$ and an ionic strength of $10^{-2} \mathrm{M}$ [10].

The samples were filled between the walls of a double walled aluminum container with $22 \mathrm{~mm}$ outer diameter and a gap of $0.05 \mathrm{~mm}$ between the walls. The same container was used consecutively for the sol, pure water, and gel samples. We note that the transfer of the samples from the glass jars to the sample container induced some shear forces on the samples. The gel sample may therefore have partly, temporarily "shear-melted" upon being filled in the sample container.

The data were treated using NATHAN (FOCUS instrument software) and DAVE (NIST neutron data analysis software [32], version 1.4, build date Oct. 18, 2007), and subsequently MATLAB (version 7.0.4.352). For the data analysis, the detectors of FOCUS were binned into 14 groups. Fits to the quasielastic neutron scattering (QENS) signal were done after interpolating the data to a regular $(Q, \omega)$ grid. Data fitting was done using the MATLAB LSQCURVEFIT algorithm.

\section{RESULTS AND DISCUSSION}

From the data recorded on a cold neutron time-of-flight (TOF) spectrometer several types of information can be ex-

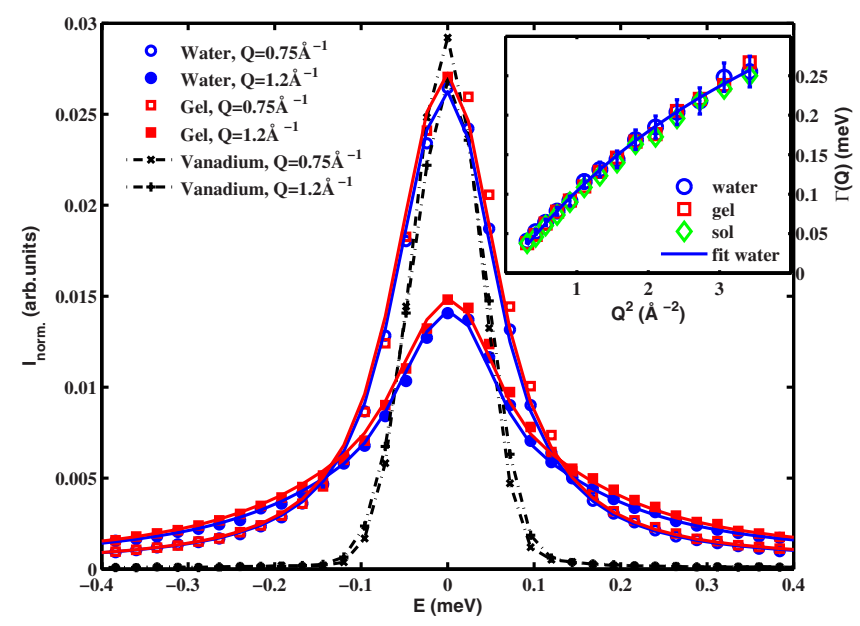

FIG. 2. (Color online) Quasielastic scattering from the water (circle symbols) and 2 wt. \% mineral gel samples (square symbols), respectively, and corresponding fits of a simple model (solid lines, see text), for two different scattering vectors $Q$ (open and filled symbols). The respective instrumental resolution measured by the scattering from vanadium is given in addition (dash-dotted lines). The fit results for the HWHM of the single Lorentzian function assumed in the model are given in the inset, completed by the result for the 1.2 wt. \% mineral sol. (Sample temperature $T=285 \mathrm{~K}$.)

tracted. The so-called QENS, i.e., the broadening in the energy transfer $E=\hbar \omega$ of the elastic line as a function of the scattering vector $Q$ due to relaxational motion in the sample, contains information on the diffusion on molecular length scales. The total scattering of the sample provides information on its scattering length density, and the inelastic scattering is characteristic of intermolecular and intramolecular vibrational modes. We discuss these types of information in the following paragraphs. The energy range and resolution of the TOF spectrometer thereby correspond to time scales of motion in the sample between approximately $40-0.4 \mathrm{ps}$. The scattering vector range translates into a length scale range probed between approximately $3 \AA$ and $30 \AA \quad(1 \AA$ $\left.=10^{-10} \mathrm{~m}\right)$. In the following paragraphs, figure captions, and labels, reference is made to the following samples: (i) a sol sample obtained by dispersing 1.2 wt. \% laponite powder in water $\left(\mathrm{H}_{2} \mathrm{O}\right)$, (ii) a gel sample $(2.0 \mathrm{wt}$. \% laponite powder in $\mathrm{H}_{2} \mathrm{O}$ ), and (iii) a pure water $\left(\mathrm{H}_{2} \mathrm{O}\right)$ sample (see Sec. II for details).

Diffusion coefficient as extracted from QENS (Fig. 2). Example QENS data and fits of a simple model are given in Fig. 2 for two scattering vectors. The model consists of the sum of a single Lorentzian line with an intensity

$$
I(Q, \omega)=I_{0} \frac{1}{\pi} \frac{\Gamma(Q)}{\hbar^{2} \omega^{2}+[\Gamma(Q)]^{2}}
$$

as a function of the amplitude $I_{0}$, energy transfer $\hbar \omega$, and scattering vector $Q$, and an elastic line (the latter taking into account the Debye-Waller factor and cryostat and sample container scattering) convoluted with the instrumental resolution. A constant and a sloped background have been added subsequent to the convolution, the latter taking a frameoverlap background into account. The half-width at half 
maximum (HWHM) $\Gamma(Q)$ of the Lorentzian line in Eq. (1) has been interpreted in terms of a simple jump diffusion model [33] and thus assumed to be

$$
\Gamma(Q)=\frac{D Q^{2}}{1+D Q^{2} \tau_{0}}
$$

with the apparent diffusion constant $D$ and the apparent residence time $\tau_{0}$. The rotational component of the diffusion has been neglected, because the rotational and translational residence times cannot be uniquely discriminated near room temperature [28]. The obtained Lorentzian linewidths from the fits according to Eq. (1) and the fits to these results according to Eq. (2) are given in the inset of Fig. 2. The error bars $\Delta \Gamma$, given in the inset for pure water only, have been obtained from the fitted function $f_{\text {fit }}$ according to $\Delta \Gamma$ $=\sqrt{\left(\partial^{2} f_{\text {fit }} / \partial^{2} \Gamma\right)^{-1}}$, i.e., from the diagonal of the error matrix of the optimum found. The apparent diffusion coefficients and residence times as extracted from the fits to our QENS data are indistinguishable for the pure water and gel samples within the accuracy of our experiment and fits. We note that in general, fit results can depend on the starting conditions and convergence criteria of the minimization algorithm. As a precaution, we have therefore applied the same starting values, boundary conditions, and convergence criteria to all individual fits of all data sets. The results from our fits are in good agreement with published values for pure water $[28,34]$, considering that we have neglected the rotational diffusion in our model. Thus from our fits we derive the apparent diffusion constants $D_{\mathrm{H}_{2} \mathrm{O}}=(1.9 \pm 0.2) \times 10^{-5} \mathrm{~cm}^{2} / \mathrm{s}$, $D_{\text {sol }}=(1.7 \pm 0.2) \times 10^{-5} \mathrm{~cm}^{2} / \mathrm{s}, \quad$ and $\quad D_{\text {gel }}=(1.8 \pm 0.2)$ $\times 10^{-5} \mathrm{~cm}^{2} / \mathrm{s}$ for the pure water, sol, and gel samples, respectively, while Gillen et al. [34] obtain for the selfdiffusion constant of pure water $D_{s, \mathrm{H}_{2} \mathrm{O}}=(1.58 \pm 0.08)$ $\times 10^{-5} \mathrm{~cm}^{2} / \mathrm{s}$ within $95 \%$ confidence limits using NMR. $D_{s, \mathrm{H}_{2} \mathrm{O}}$ agrees nearly with the translational diffusion constant $D_{T, \mathrm{H}_{2} \mathrm{O}}=1.57 \times 10^{-5} \mathrm{~cm}^{2} / \mathrm{s}$ which can be calculated from the results reported in Ref. [28] for $T=12{ }^{\circ} \mathrm{C}$. The apparent residence times resulting from our fits are $\tau_{0, \mathrm{H}_{2} \mathrm{O}}=1.0 \mathrm{ps}, \tau_{0, \text { sol }}$ $=1.0 \mathrm{ps}$, and $\tau_{0, \text { gel }}=0.9 \mathrm{ps}$, which is reasonably near the value of $1.66 \mathrm{ps}$ reported in Ref. [28] for pure water at $T$ $=12{ }^{\circ} \mathrm{C}$, given that our errors for the residence time are on the same order of magnitude as the fitted value. Since the measured signal has most accurately to be assumed to be a convolution of the rotational and translational components, the Lorentzian width $\Gamma(Q)$ in Eq. (1) is actually the sum of the rotational and translational components. Therefore, our fit overestimates the translational linewidth, which is in agreement with the discrepancy between our fit results and the literature values.

Elastic scattering and total scattering (Fig. 3). Figure 3 provides an overview of the elastic and total scattering contributions in the energy range dominated by elastic and quasielastic scattering. From Fig. 3 (bottom) it becomes evident that most of the scattering is quasielastic and not elastic for both the sol and the gel samples. Thus without further consideration it can be inferred from this figure alone that the sol and gel samples behave similar to liquids and unlike solids over the accessible scattering vector range of the ex-
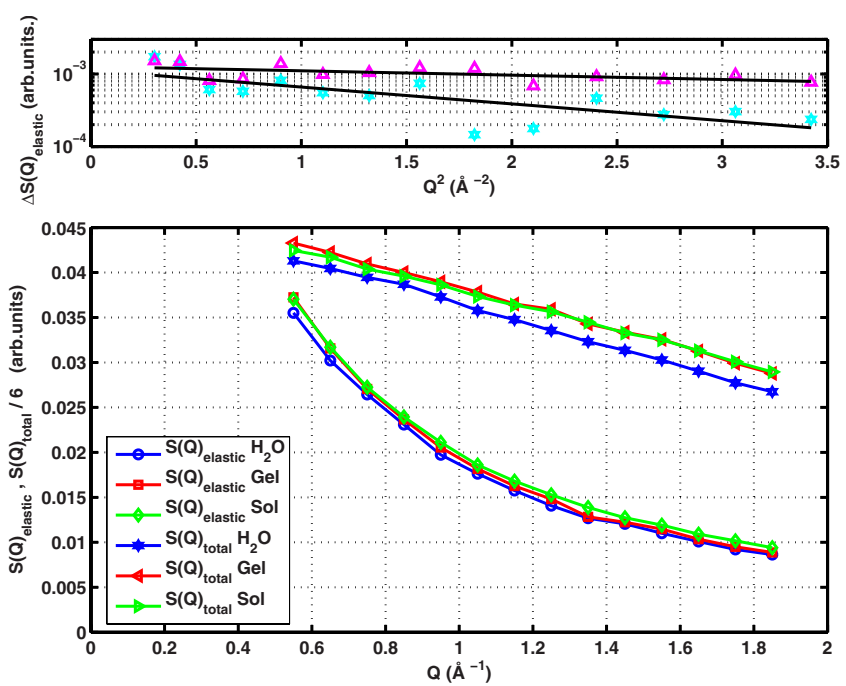

FIG. 3. (Color online) Bottom: Elastic scattering $S(Q, \omega=0)$ obtained from the acquisition channel centered at $\omega=0$ (lower three lines) and total scattering $S(Q)=\sum_{E_{\min }}^{E_{\max }} S(Q, \omega) d \omega$ (upper three lines, $E_{\min }=-1.2 \mathrm{meV}, E_{\max }=0.6 \mathrm{meV}$ ) after interpolation of the data to an $\omega$ axis with equidistant points separated by $\Delta(\hbar \omega)=0.024 \mathrm{meV}$. Note that the sample container and cryostat scattering have not been subtracted and that the total scattering has been divided by 6 for better visibility in the same plot (see $y$ axis label). (Sample temperature $T=285 \mathrm{~K}$.) Top: Difference signals of the elastic scattering gel- $\mathrm{H}_{2} \mathrm{O}$ (asterisks), and sol- $\mathrm{H}_{2} \mathrm{O}$ (triangles), respectively. The lines are fits according to $\Delta S(Q)_{\text {elastic }} \propto \exp \left(-\left\langle u^{2}\right\rangle Q^{2}\right)$ with slope $\left\langle u^{2}\right\rangle$.

periment. This observation complements the information discussed along with Fig. 2. The sol and gel samples have a slightly higher mass density than the pure water sample due to the added minerals (see Sec. II for measured values). This contributes to the increased total scattering (Fig. 3, bottom). The sum of the scattering over all detectors and time-of-flight channels $I_{\text {total }}$ gives the following ratios: $I_{\text {total, gel }} / I_{\text {total }, \mathrm{H}_{2} \mathrm{O}}=1.06$ and $I_{\text {total, sol }} / I_{\text {total }, \mathrm{H}_{2} \mathrm{O}}=1.05$. Note that the neutron scattering experiment is sensitive to the scattering length density rather than the mass density of the sample. The elastic scattering is also somewhat increased in both the sol and the gel compared to pure water (Fig. 3, bottom). The differences of the elastic scattering, gel, and pure water signal, and sol and water signal, respectively, are small (Fig. 3, top). Due to the mixture of quasielastic scattering at zero energy transfer and true elastic scattering, their $Q$ dependence cannot be uniquely identified with a thermal DebyeWaller factor due to underdamped vibrational motions. A steeper slope $\left\langle u^{2}\right\rangle$ of a Debye-Waller-type factor $\propto \exp \left(-\left\langle u^{2}\right\rangle Q^{2}\right)$ (lines in Fig. 3, top) in the gel could indicate more pronounced vibrational motions in the gel compared to the sol. However, the accuracy of the difference spectra is insufficient for a further discussion. We note that even the minute elastic scattering may be only an apparent elastic scattering and that at a sufficiently high energy-resolution, no true elastic scattering from the sample might remain.

TOF difference spectra (Figs. 4 and 5). The low-energy inelastic scattering signals from the pure water, sol, and gel samples and the difference spectra are given in Figs. 4 and 5. The low-energy inelastic features visible in the time-of-flight 


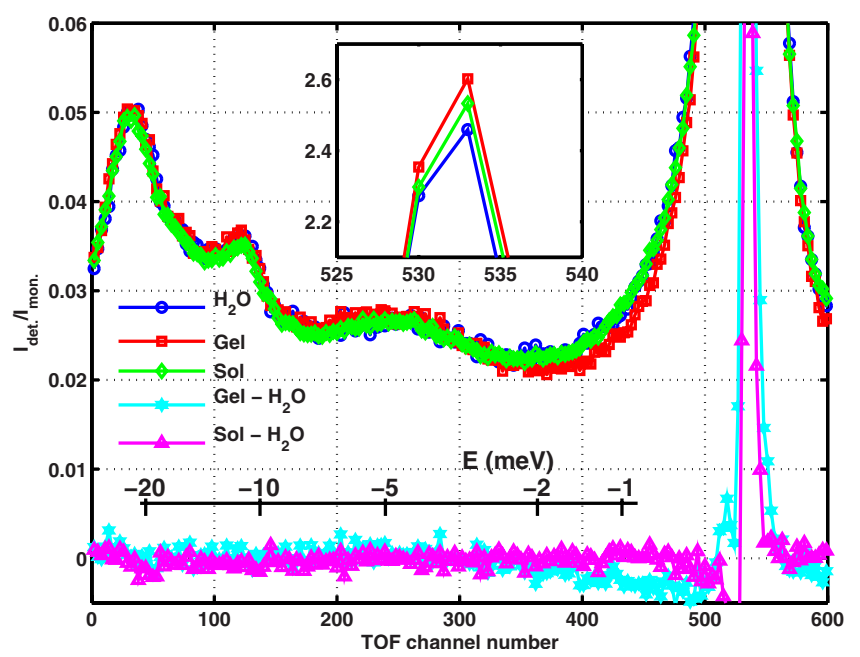

FIG. 4. (Color online) Intensity vs time-of-flight (TOF) channel number (including sample container scattering, TOF channel width $5 \mu \mathrm{s})$ and difference spectra as specified in the legend. The intensities of the 60 detectors at the lowest scattering angles have been summed (average elastic scattering vector $Q \approx 0.65 \AA^{-1}$ ). The additional energy axis indicates the calculated energy transfer. The inset displays the region near the elastic line using the same axis units as the main part of the figure. (Sample temperature $T=285 \mathrm{~K}$.)

spectra may be identified with the results known since long for $\mathrm{H}_{2} \mathrm{O}$ [35]. They are due to different types of hindered motions of the water molecules. Note that the spectra are affected by aluminum phonons at large energy transfers (above $|\Delta E|=15 \mathrm{meV}$ ), since the container scattering is not subtracted. There is no apparent difference in the inelastic scattering from water and gel. Only an additional elastic scattering contribution in the sol and gel samples becomes evident in the difference spectra (see also Fig. 3). This can be explained by both the small scattering contribution of the added minerals themselves and interfacial water immobilized

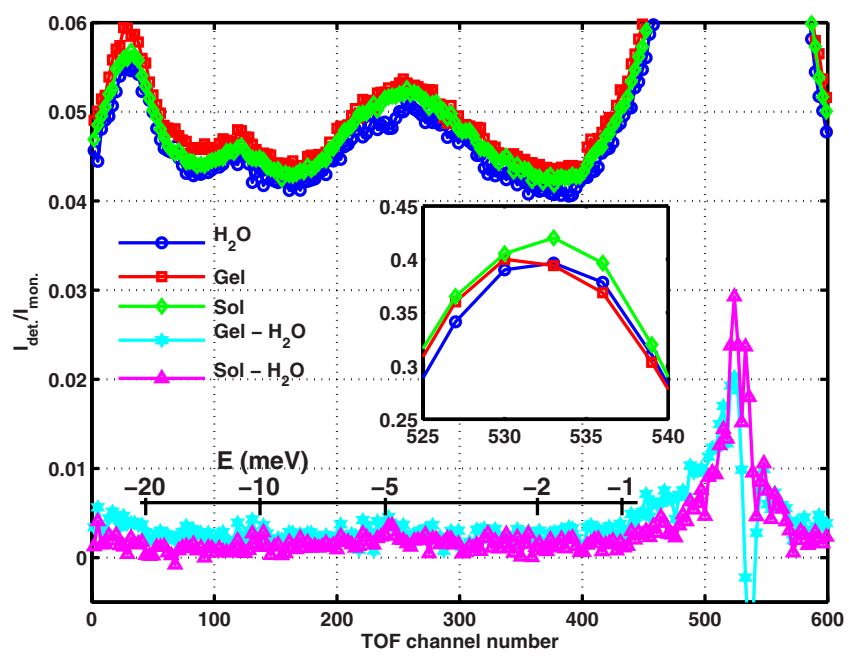

FIG. 5. (Color online) Intensity vs TOF channel number (including sample container scattering) and difference spectra analogously to Fig. 4 for the summed intensities of the 60 detectors at the highest scattering angles (average elastic scattering vector $Q$ $\approx 1.75 \AA^{-1}$ ). (Sample temperature $T=285 \mathrm{~K}$.) on the minerals. One might speculate about a minute broadening of the excess elastic scattering at high $Q$ (Fig. 5). This could be due to diffusive (for instance, rotational) motions of water bound to the laponite particles.

Summarizing our observations, we see no significant change in the water mobility in the sol and gel samples as compared to pure water in a scattering vector range of approximately $0.2 \AA^{-1} \leqslant Q \leqslant 2 \AA^{-1}$ and an energy transfer range of approximately $100 \mu \mathrm{eV} \leqslant \omega \leqslant 20 \mathrm{meV}$ corresponding to picosecond time scales of diffusive motions. This equally holds for nanosecond time scales at similar scattering vectors accessible by neutron backscattering spectrometers [15]. Note that this does not rule out that there may be changes at smaller scattering vectors not accessible in our experiments corresponding to larger (mesoscopic) length scales (see the light scattering and XPCS work cited in the Introduction). Our observation furthermore does not rule out that a small proportion of the water molecules in the sol and gel samples may be immobilized by being bound to the mineral surfaces. However, their share is too small to significantly alter the resulting average mobility that can be derived from the spectra. A better quantification of the minute effects of water bound to the laponite minerals would require significantly better counting statistics and thus more neutron beam time than available for this type of study, where the acquisition time per sample was restricted to be on the order of half a day. In the context of the general interpretation of results concerning hydrogels, we note that some of their properties may depend on their history on a time scale of several days or even months, as discussed in the Introduction in the context of existing literature.

\section{CONCLUSIONS}

In agreement with the observation reported in Ref. [4] our results are commensurate with the assumption that the atomic-scale dynamical properties of intracellular water, i.e., in our model system "intracompartment" water, are essentially the properties of bulk water. Hence, on length scales smaller than the assumed compartment dimensions in the house-of-cards model of the gel, there is no significant change of the water mobility. The change of the macroscopic mechanical and rheological properties at the sol-gel transition is therefore not reflected by any corresponding significant change of the dynamical properties of most of the water at intermolecular and intramolecular length scales. Importantly, we therefore also conclude alluding to biological contexts that the observation of unaffected atomic-scale water mobility in intracellular environments does not contradict the gel or colloidal model of a cell. Rather, simple model gels yield the same observations when probed by neutron spectroscopy. The gel- or colloidal model of a living cell cannot be assumed to be refuted by the neutron spectroscopic results available at present. In this respect our conclusion might add a somewhat different perspective to the interpretions of the results obtained from a real biological system as reported in Ref. [4]. However, we emphasize that any generalization of conclusions has to be taken with caution and that biological systems evidently have different characteristics than our model system. 
Our conclusion put in other words, both in our simple model system of a clay mineral hydrogel as well as in much more complex living cells water is macroscopically immobilized. The water diffusion on interatomic length scales is not or nearly not influenced by this macroscopic arrest. Water may thus be macroscopically immobilized without significantly affecting its microscopic diffusion which is essential for its biological role. The nonergodic property of a hydrogel [24] may in this respect only concern the gelator and not the water. By contrast, macroscopic transport processes involving large volume elements, such as convection currents, are suppressed in a gel-like material. In the case of our model system of a hydrogel, the macroscopic arrest of the water takes place without the presence of a surrounding "cell" membrane.

Our conclusion holds notwithstanding the minute effects of water bound to the mineral particles in the suspension, and by the suspended particles themselves. A small proportion of immobilized water is analogously discussed in the context of biological systems [4]. A more quantitative understanding of this small scattering contribution will require significantly more neutron beam time or higher brilliance neutron beams to obtain more accurate difference spectra.

\section{ACKNOWLEDGMENTS}

We thank Matthias Elender (PSI, Switzerland) for designing the sample container for the experiment and technical support. We are indebted to János Major (MPI-MF Stuttgart, Germany) for many stimulating discussions. L.W. acknowledges support from the MPI-MF in 2002 permitting the experiments presented in the supplementary online material (Figs. 1-3 [15]), to be carried out at the ILL. We are grateful to Pierre Terech (CEA Grenoble, France) for rheology measurements (supplementary online material [15], Fig. 5). This work is based on experiments performed at the Swiss spallation neutron source SINQ, Paul Scherrer Institute, Villigen, Switzerland. The spectrometer FOCUS has been funded by the German ministry BMBF. Complementary experiments have been performed using neutron beams of the ILL.
[1] P. Ball, Chem. Rev. (Washington, D.C.) 108, 74 (2008).

[2] J. T. Trevors and G. H. Pollack, Prog. Biophys. Mol. Biol. 89, 1 (2005).

[3] A. Y. Grosberg, T. T. Nguyen, and B. I. Shoklovskii, Rev. Mod. Phys. 74, 329 (2002).

[4] M. Jasnin, M. Moulin, M. Haertlein, G. Zaccai, and M. Tehei, EMBO Rep. 9, 543 (2008).

[5] M. Tehei, B. Franzetti, K. Wood, F. Gabel, E. Fabiani, M. Jasnin, M. Zamponi, D. Oesterhelt, G. Zaccai, M. Ginzburg, and B. Z. Ginzburg, Proc. Natl. Acad. Sci. U.S.A. 104, 766 (2007).

[6] E. Persson and B. Halle, Proc. Natl. Acad. Sci. U.S.A. 105, 6266 (2008).

[7] E. Del Gado, A. Fierro, L. de Arcangelis, and A. Coniglio, Europhys. Lett. 63, 1 (2003).

[8] E. Zaccarelli, J. Phys.: Condens. Matter 19, 323101 (2007).

[9] H. Van Olphen, Discuss. Faraday Soc. 11, 82 (1951).

[10] A. Mourchid, A. Delville, J. Lambard, E. Lecolier, and P. Levitz, Langmuir 11, 1942 (1995).

[11] H. K. Schmidt, E. Geiter, M. Mennig, H. Krug, C. Becker, and R. P. Winkler, J. Sol-Gel Sci. Technol. 13, 397 (1998).

[12] C. J. Brinker and G. W. Scherer, Sol-Gel Science (Academic Press, San Diego, 1990).

[13] http://www.laponite.com

[14] J. D. F. Ramsay and P. Lindner, J. Chem. Soc., Faraday Trans. 89, 4207 (1993).

[15] See EPAPS Document No. E-PLEEE8-78-051812 for supplementary figures. For more information on EPAPS, see http:// www.aip.org/pabservs/epaps.html.

[16] B. Struth, F. Rieutord, O. Konovalov, G. Brezesinski, G. Grübel, and P. Terech, Phys. Rev. Lett. 88, 025502 (2001).

[17] B. Struth, A. Vorobiev, T. Seydel, L. Wiegart, and J. Major, Physica B 350, E917 (2004).

[18] M. Dijkstra, J. P. Hansen, and P. A. Madden, Phys. Rev. E 55,
3044 (1997).

[19] C. Martin, F. Pignon, J. M. Piau, A. Magnin, P. Lindner, and B. Cabane, Phys. Rev. E 66, 021401 (2002).

[20] L. J. Michot, I. Bihannic, S. Maddi, S. S. Funari, C. Baravian, P. Levitz, and P. Davidson, Proc. Natl. Acad. Sci. U.S.A. 103, 16101 (2006).

[21] P. Porion, S. Rodts, M. Al-Mukhtar, A. M. Faugère, and A. Delville, Phys. Rev. Lett. 87, 208302 (2001).

[22] B. Ruzicka, L. Zulian, and G. Ruocco, Phys. Rev. Lett. 93, 258301 (2004)

[23] R. Bandyopadhyay, D. Liang, H. Yardimci, D. A. Sessoms, M. A. Borthwick, S. G. J. Mochrie, J. L. Harden, and R. L. Leheny, Phys. Rev. Lett. 93, 228302 (2004).

[24] B. Ruzicka, L. Zulian, R. Angelini, M. Sztucki, A. Moussaïd, and G. Ruocco, Phys. Rev. E 77, 020402(R) (2008).

[25] http://spectroscopy.web.psi.ch/focus/

[26] B. Ruzicka, L. Zulian, and G. Ruocco, Langmuir 22, 1106 (2006).

[27] J. Teixeira, M. C. Bellissent-Funel, and S. H. Chen, J. Phys.: Condens. Matter 2, SA105 (1990).

[28] J. Teixeira, M. C. Bellissent-Funel, S. H. Chen, and A. J. Dianoux, Phys. Rev. A 31, 1913 (1985).

[29] S. Mitra, R. Mukhopadhyay, I. Tsukushi, and S. Ikeda, J. Phys.: Condens. Matter 13, 8455 (2001).

[30] L. Li, L. Harnau, S. Rosenfeldt, and M. Ballauff, Phys. Rev. E 72, 051504 (2005).

[31] http://www.scprod.com/pdfs/LaponiteBrochureE.pdf

[32] http://www.ncnr.nist.gov/dave/

[33] P. A. Egelstaff An Introduction to the Liquid State, 2nd ed. (Clarendon Press, Oxford, 1994).

[34] K. T. Gillen, D. C. Douglas, and M. J. R. Hoch, J. Chem. Phys. 57, 5117 (1972).

[35] D. J. Hughes, H. Palevsky, W. Kley, and E. Tunkelo, Phys. Rev. Lett. 3, 91 (1959). 